\author{
S.-W. BAHK ${ }^{1, *,}$ \\ P. ROUSSEAU ${ }^{2}$ \\ T.A. PLANCHON ${ }^{3}$ \\ V. CHVYKOV ${ }^{2}$ \\ G. KALINTCHENKO ${ }^{2}$ \\ A. MAKSIMCHUK ${ }^{2}$ \\ G.A. MOUROU ${ }^{4}$ \\ V. YANOVSKY ${ }^{2}$
}

\section{Characterization of focal field formed by a large numerical aperture paraboloidal mirror and generation of ultra-high intensity $\left(10^{22} \mathrm{~W} / \mathrm{cm}^{2}\right)$}

${ }^{1}$ Laboratory for Laser Energetics, University of Rochester, Rochester, NY 14623, USA

${ }^{2}$ FOCUS Center and Center for Ultrafast Optical Science, University of Michigan, Ann Arbor, MI 48109, USA

${ }^{3}$ Department of Physics, Colorado School of Mines, Golden, CO 80401, USA

${ }^{4}$ Laboratoire d'Optique Appliquée, ENSTA, Ecole Polytechnique, 91761 Palaiseau Cedex, France

\section{Received: 21 September 2004 / \\ Revised version: 9 March 2005 \\ Published online: 3 May 2005 • () Springer-Verlag 2005}

ABSTRACT We describe a method to measure the aberrations of a high numerical aperture off-axis paraboloid and correct for the aberrations using adaptive optics. It is then shown that the characterized aberrations can be used to accurately calculate the electromagnetic field at the focus using the Stratton$\mathrm{Chu}$ vector diffraction theory. Using this methodology, an intensity of $7 \times 10^{21} \mathrm{~W} / \mathrm{cm}^{2}$ was demonstrated by focusing a 45-TW laser beam with an $f / 0.6,90^{\circ}$ off-axis paraboloid after correcting the aberrations of the paraboloid and the lowenergy reference beam. The intensity can be further increased to $1 \times 10^{22} \mathrm{~W} / \mathrm{cm}^{2}$ by including in the correction algorithm the wavefront difference between the reference beam and the high-energy beam.

PACS 42.25.Fx; 41.20.Jb; 42.15.Fr rate and low-amplitude fluctuations. In this article we present a method with which we can directly measure the aberrations from high-NA optics. With this measurement, we were able to correct for the aberrations using a deformable mirror and could achieve a Strehl ratio of 0.9. The measured near field not only leads to the benefit of adaptive optics but also to the possibility of characterizing the whole field at the focus using a diffraction integral. For this purpose, we first develop far-field vector diffraction formulae suitable for an off-axis paraboloid in Sect. 2, which are necessary for the calculation of high-NA focusing. Some points are then made clear in Sect. 3 concerning the imaging of the field before proceeding to the experimental part of this article, since it is needed to justify our imaging method. In the experimental part as presented in Sects. 4 and 5 , the aberration-measurement setup and the correction algorithm are presented for an $f / 0.6,90^{\circ}$ off-axis paraboloid and this method is applied to generate an extreme power density of $7 \times 10^{21} \mathrm{~W} / \mathrm{cm}^{2}$ using a 45 -TW laser beam. Overall, the article presents all the formulae and details that could not be listed for want of space in our previous publication [2].

2

\section{Vector diffraction formulae \\ for an off-axis paraboloid}

In this section, we derive an expression for the diffracted field from an off-axis paraboloid. Our diffraction formulae are based on the Stratton-Chu theory. The geometry of the paraboloid and the angle of incidence are drawn in Fig. 1. To derive a vector field near the focus, we use the Stratton-Chu formula [3]

$$
\begin{aligned}
\mathbf{E}(Q)= & \frac{1}{4 \pi} \iint[\mathrm{i} k \eta(\mathbf{n} \times \mathbf{H}) G \\
& +(\mathbf{n} \times \mathbf{E}) \times \nabla G+(\mathbf{n} \cdot \mathbf{E}) \nabla G] \mathrm{d} A, \\
\mathbf{H}(Q)= & \frac{1}{4 \pi} \iint\left[\mathrm{i} k \frac{1}{\eta}(\mathbf{E} \times \mathbf{n}) G\right. \\
& +(\mathbf{n} \times \mathbf{H}) \times \nabla G+(\mathbf{n} \cdot \mathbf{H}) \nabla G] \mathrm{d} A,
\end{aligned}
$$

Fax: 585-275-5960, E-mail: sbah@lle.rochester.edu

* Present address: Laboratory for Laser Energetics, University of Rochester, 250 East River Road, Rochester, NY 14623, USA 


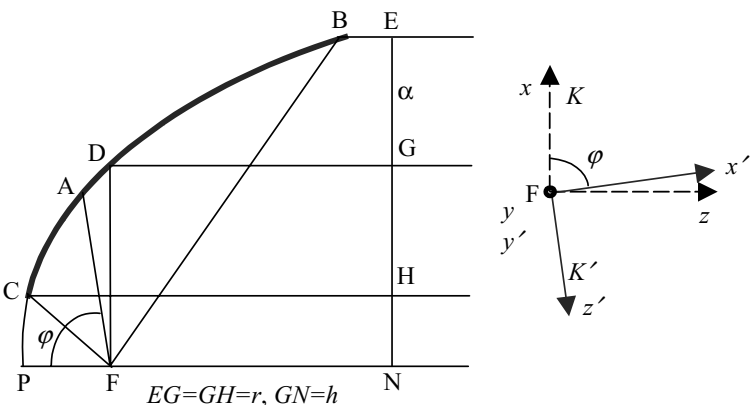

FIGURE 1 Geometry of paraboloid reflection

where $G=\exp \left(\mathrm{i} k r_{\mathrm{SQ}}\right) / r_{\mathrm{SQ}} . S$ is a point on the surface of the paraboloid and $Q$ is a point near the focus. $\mathbf{n}$ is a unit vector normal to the paraboloid surface and the incremental area $\mathrm{d} A$ is also taken across the surface. $\eta$ is the intrinsic impedance in free space $\left(\sqrt{\mu_{0} / \epsilon_{0}}\right)$ and the units used throughout this article are SI. The contour integral in the original Stratton-Chu formula was not included, because it is negligible for the far-field calculation. The field component inside the integral is to be considered as the sum of the incident and reflected fields. The totality of the field can be expressed in terms of only the incident field [3]:

$\mathbf{E}(S)=\mathbf{E}_{\mathrm{i}}+\mathbf{E}_{\mathrm{r}}=2 \mathbf{n}\left[\mathbf{n} \cdot \mathbf{E}_{\mathrm{i}}(S)\right]$,

$\mathbf{H}(S)=\mathbf{H}_{\mathrm{i}}+\mathbf{H}_{\mathrm{r}}=2 \mathbf{H}_{\mathrm{i}}(S)-2 \mathbf{n}\left[\mathbf{n} \cdot \mathbf{H}_{\mathrm{i}}(S)\right]$.

With these relations, the second term of Eq. (1) and the first and third terms of Eq. (2) vanish.

The paraboloidal surface is given as

$z=\frac{x^{2}+y^{2}}{4 f}-f \equiv s f-f$,

where $f$ is the parent focal length, and the usable area of the paraboloid is specified as

$\Sigma:(x-h)^{2}+y^{2} \leq r^{2}$,

where $r$ is the radius of the paraboloid and $h$ is the distance from the $z$ axis to the center of the incoming beam. The normal vector to this paraboloidal surface and the infinitesimal area is

$$
\hat{\mathbf{n}}=\frac{1}{\sqrt{1+s}}\left(-\frac{x}{2 f} \hat{\mathbf{x}}-\frac{y}{2 f} \hat{\mathbf{y}}+\hat{\mathbf{z}}\right),
$$

$\mathrm{d} A=\sqrt{1+s} \mathrm{~d} x \mathrm{~d} y$.

As denoted in Fig. 1, we use a tilted frame $K^{\prime}$ for focal coordinates and the frame $K$ for the incident beam and the paraboloidal surface. The rotation angle $\varphi$ is defined as $\angle A F P$, where point $A$ is fixed such that $\angle A F C=\angle A F B$. Note that the line $\overline{A F}$ does not coincide with the line $(\overline{D F})$ connected to the center line of the incoming beam. We call the line $\overline{A F}$ the middle ray and the line $\overline{D F}$ the center ray. We chose the middle ray as the alignment axis because it not only gives a symmetric range of direction cosines but also at this angle the intensity of the longitudinal field at the center is minimized. We denote the coordinates for each frame as $\left(x^{\prime}, y^{\prime}, z^{\prime}\right)$ and $(x, y, z)$, respectively. Thus, the coordinates for $Q,\left(x^{\prime}, y^{\prime}, z^{\prime}\right)$ in the $K^{\prime}$ frame, are transformed to $\left(x^{\prime} \cos \varphi-\right.$ $z^{\prime} \sin \varphi, y^{\prime}, x^{\prime} \sin \varphi+z^{\prime} \cos \varphi$ ) in the $K$ frame. To calculate the diffraction of monochromatic light, we assume that the incoming beam does not have longitudinal components for both electric and magnetic fields. Although this assumption is mathematically inconsistent, for beam sizes much larger than the wavelength, one can safely neglect the longitudinal component. The assumed incident field defined on the plane $\alpha$ (see Fig. 1) with the time-oscillation term dropped is given as

$\mathbf{E}_{\mathrm{i} \alpha}=\left(\mathcal{E}_{0 \mathrm{x}} \hat{\mathbf{x}}+\mathcal{E}_{0 \mathrm{y}} \hat{\mathbf{y}}\right) \exp (-\mathrm{i} k z)$,

$\mathbf{H}_{\mathrm{i}, \alpha}=\frac{1}{\eta}\left(\mathcal{E}_{0 \mathrm{y}} \hat{\mathbf{x}}-\mathcal{E}_{0 \mathrm{x}} \hat{\mathbf{y}}\right) \exp (-\mathrm{i} k z)$,

where $z$ belongs to the position of the plane $\alpha$. To find the incident field on the paraboloidal surface, one needs to multiply additional phase from the plane $\alpha$ to the surface assuming that the diffraction effect is negligible. The plane $\alpha$ has to be located close enough to the surface, or the Fresnel number for this propagation has to be large, due to this assumption. Let us call the point on the plane $\alpha S^{\prime}$. The phase term belonging to the Green function in the integral, calculating the distance from $S^{\prime}$ to $S$ and then to $Q$, can then be expressed as

$$
\begin{aligned}
\mathrm{i} k \phi= & -\mathrm{i} k\left(x^{\prime} \frac{x \cos \varphi+z \sin \varphi}{f(1+s)}+y^{\prime} \frac{y}{f(1+s)}\right. \\
& \left.+z^{\prime} \frac{-x \sin \varphi+z \cos \varphi}{f(1+s)}\right)
\end{aligned}
$$

dropping insignificant constant-phase terms. Quadratic terms of focal field variables were also dropped under the condition that $\left(x^{\prime 2}+y^{\prime 2}+z^{\prime 2}\right) \ll 2 f(1+s)\left|\left(x^{\prime} p+y^{\prime} q+z^{\prime} m\right)\right|$ (for $(p, q, m)$, refer to equations below). The meaning of the terms in the phase attached to the primed coordinates is as follows. If one tries to map the coordinates of the incident beam, $(x, y)$, on to a plane which is perpendicular to the propagation direction of the focusing beam and placed arbitrarily some distance $(L)$ away from the focus, one obtains new coordinates

$(X, Y)=\left(L \cdot \frac{p}{m}, L \cdot \frac{q}{m}\right)$,

where $p, q$, and $m$ are the direction cosines for $(X, Y, L)$ and can be identified exactly as the terms in the above expression, which are

$$
\begin{aligned}
& p=-\frac{(x \cos \varphi+z \sin \varphi)}{f(1+s)}, \\
& q=-\frac{y}{f(1+s)},
\end{aligned}
$$

$m=-\frac{(-x \sin \varphi+z \cos \varphi)}{f(1+s)}=\sqrt{1-p^{2}-q^{2}}$.

Thus, the use of the direction cosines automatically includes the effect of the image distortion after paraboloidal reflection. Figure 2 shows typical image distortion after paraboloidal reflection. An inversion formula from $(p, q, m)$ to $(x, y, z)$ is as follows.

$$
\begin{aligned}
& x=\frac{2 f(-p \cos \varphi+m \sin \varphi)}{(1+p \sin \varphi+m \cos \varphi)}, \\
& y=\frac{2 f(-q)}{(1+p \sin \varphi+m \cos \varphi)},
\end{aligned}
$$



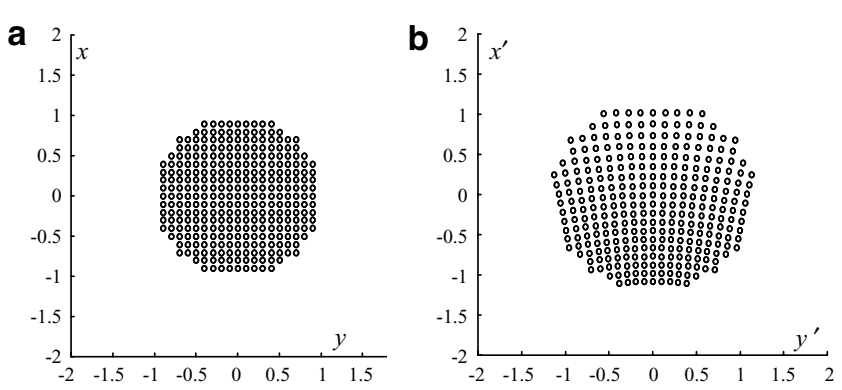

FIGURE 2 Image distortion before (a) and after (b) reflection off a paraboloid $\left(60^{\circ}\right.$, effective $\left.f / \#=2\right)$

$$
z=\frac{2 f(-p \sin \varphi-m \cos \varphi)}{(1+p \sin \varphi+m \cos \varphi)}
$$

To express the integral in terms of direction cosines, we transform the incremental area $\mathrm{d} x \mathrm{~d} y$ into $\partial(x, y) / \partial(p, q) \mathrm{d} p \mathrm{~d} q \equiv J \mathrm{~d} p \mathrm{~d} q$, where

$$
J=\frac{4 f^{2}\left\{(p+\sin \varphi)^{2}+(m+\cos \varphi)^{2}-(p \cos \varphi-m \sin \varphi)^{2}\right\}}{m(1+p \sin \varphi+m \cos \varphi)^{4}} .
$$

The Jacobian can be used to calculate the inhomogeneity of reflected image intensity. Using the relations given above, the main integral can be summarized as

$$
\begin{aligned}
E_{\mathrm{x}}= & \frac{\mathrm{i}}{\lambda} \iint\left[\mathcal{E}_{0 \mathrm{x}}\left(\frac{1}{f(1+s)}-\frac{x^{2}}{2 f^{3}(1+s)^{2}}\right)\right. \\
& \left.+\mathcal{E}_{0 \mathrm{y}}\left(\frac{-x y}{2 f^{3}(1+s)^{2}}\right)\right] \mathrm{e}^{\mathrm{i} k \phi} J \mathrm{~d} p \mathrm{~d} q, \\
E_{\mathrm{y}}= & \frac{\mathrm{i}}{\lambda} \iint\left[\mathcal{E}_{0 \mathrm{x}}\left(\frac{-x y}{2 f^{3}(1+s)^{2}}\right)\right. \\
& \left.+\mathcal{E}_{0 \mathrm{y}}\left(\frac{1}{f(1+s)}-\frac{y^{2}}{2 f^{3}(1+s)^{2}}\right)\right] \mathrm{e}^{\mathrm{i} k \phi} J \mathrm{~d} p \mathrm{~d} q,
\end{aligned}
$$

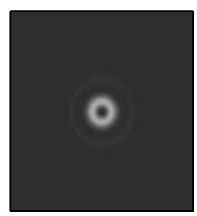

$\left|E_{x}\right|^{2}$

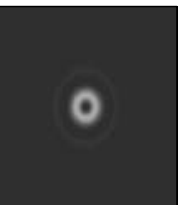

$\left|E_{x}\right|^{2}$

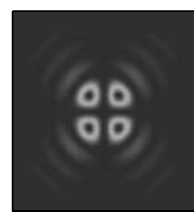

$\left|E_{y}\right|^{2}$

a

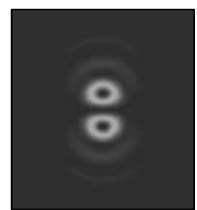

$\left|E_{y}\right|^{2}$

c

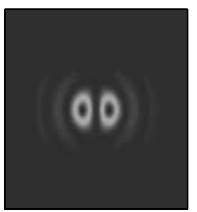

$\left|E_{z}\right|^{2}$

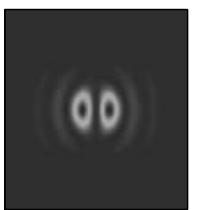

$\left|E_{z}\right|^{2}$

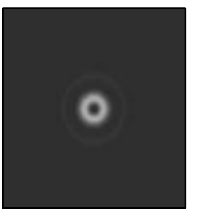

$\left|E_{x}\right|^{2}$

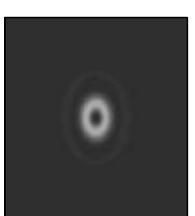

$\left|E_{x}\right|^{2}$
$E_{\mathrm{z}}=\frac{\mathrm{i}}{\lambda} \iint\left(x \mathcal{E}_{0 \mathrm{x}}+y \mathcal{E}_{0 \mathrm{y}}\right) \frac{1}{f^{2}(1+s)^{2}} \mathrm{e}^{\mathrm{i} k \phi} J \mathrm{~d} p \mathrm{~d} q$,

$H_{\mathrm{x}}=\frac{\mathrm{i}}{\lambda} \iint \frac{1}{\eta f^{2}(1+s)^{2}}$

$\times\left[-\frac{x y}{2 f} \mathcal{E}_{0 \mathrm{x}}+\left(s f-f-\frac{y^{2}}{2 f}\right) \mathcal{E}_{0 \mathrm{y}}\right] \mathrm{e}^{\mathrm{i} k \phi} J \mathrm{~d} p \mathrm{~d} q$,

$H_{\mathrm{y}}=\frac{\mathrm{i}}{\lambda} \iint \frac{1}{\eta f^{2}(1+s)^{2}}$

$\times\left[\left(\frac{x^{2}}{2 f}-s f+f\right) \mathcal{E}_{0 \mathrm{x}}+\frac{x y}{2 f} \mathcal{E}_{0 \mathrm{y}}\right] \mathrm{e}^{\mathrm{i} k \phi} J \mathrm{~d} p \mathrm{~d} q$,

$H_{\mathrm{z}}=\frac{\mathrm{i}}{\lambda} \iint \frac{1}{\eta f^{2}(1+s)^{2}}\left(y \mathcal{E}_{0 \mathrm{x}}-x \mathcal{E}_{0 \mathrm{y}}\right) \mathrm{e}^{\mathrm{i} k \phi} J \mathrm{~d} p \mathrm{~d} q$,

$\phi=x^{\prime} p+y^{\prime} q+z^{\prime} m$.

To obtain field values in the reference frame $K^{\prime}$, the following rotation is required.

$E_{\mathrm{x}}^{\prime}=E_{\mathrm{x}} \cos \varphi+E_{\mathrm{z}} \sin \varphi$,

$E_{\mathrm{y}}^{\prime}=E_{\mathrm{y}}$

$E_{\mathrm{z}}^{\prime}=-E_{\mathrm{x}} \sin \varphi+E_{\mathrm{z}} \cos \varphi$.

The same rotation is applied to the $H$-field.

These expressions for the $E^{\prime}\left(x^{\prime}, y^{\prime}, z^{\prime}\right)$ and $H^{\prime}\left(x^{\prime}, y^{\prime}, z^{\prime}\right)$ fields satisfy all the four Maxwell equations in vacuum with respect to the reference frame $K^{\prime}$. Note that the magnetic field, $\mathbf{H}^{\prime}$, was derived not from $\mathbf{E}^{\prime}$ but directly from the integral of Eq. (10), although it turns out that the results are the same. Examples of vector field calculations are shown in Fig. 3 using incident polarization along the $x$ axis.

The variables for the field envelope inside the integral are now $p$ and $q$. To avoid confusion with existing focal field diffraction formulae, we note that, although the envelope functions are expressed in terms of direction cosines, they are not Fourier-transformed quantities of $\mathcal{E}_{0}(x, y)$. They are rather based on $\mathcal{E}_{0}(x(p, q), y(p, q))$. The spectral component used in $[4,5]$ is the Fourier-transformed quantity of
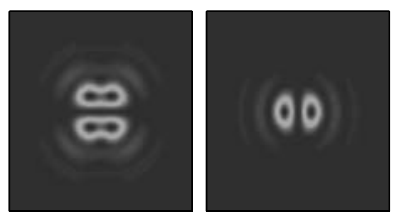

$\left|E_{y}\right|^{2}$

$\left|E_{z}\right|^{2}$

b
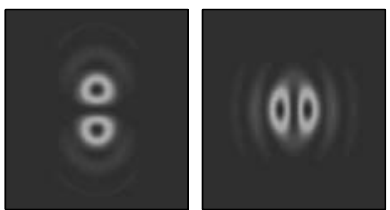

$\left|E_{y}\right|^{2}$

$\left|E_{z}\right|^{2}$

d

FIGURE 3 Focal intensity distribution at $\mathbf{a} 0^{\circ}, \mathbf{b ~} 10^{\circ}, \mathbf{c} 45^{\circ}$, and $\mathbf{d} 90^{\circ}$ incidence. $f / \#=1$ in all cases and incident polarization is along the $x$ axis 


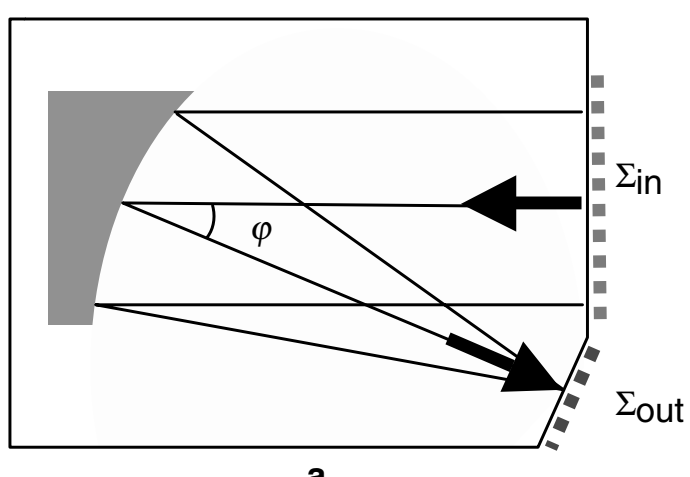

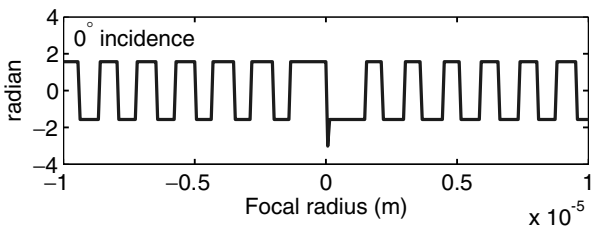

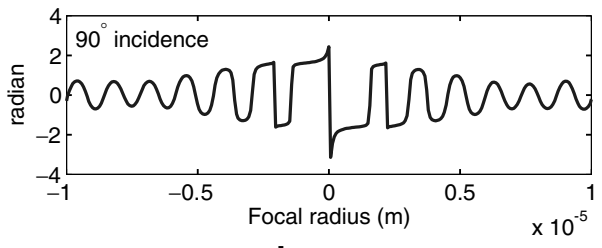

b

FIGURE 4 a Enclosure geometry for calculating energy-momentum conservation. b Phase difference between longitudinal field and transverse field ( $x$ ) with on-axis focusing (upper) and with off-axis focusing (lower). Numerical apertures in (b) were kept the same at 0.37

the field at the focus. The authors in these works begin from an assumed field shape (Gaussian) at the focus and arrive at higher-order field corrections satisfying the vanishing of field divergence, whereas we begin from the incident field before focusing and derive the field near the focus. The formulae obtained in this section provide a more practical approach since one does not have to assume the zeroth-order form of the field at the focus and the calculation can be performed with the fast Fourier transform (FFT) algorithm without involving complicated mathematical functions.

\subsection{Accuracy of the theory}

The accuracy of the vector diffraction formulae presented in this article can be tested with two criteria. The first criterion is that they have to satisfy all Maxwell's equations, which was already shown. The second criterion is the energy-momentum conservation. The conservation method was presented in our recent conference paper using on-axis geometry; we here generalize the same method to the offaxis case [6]. If one applies the complex Poynting theorem [7] to the harmonic fields surrounded by a hypothetical enclosure (Fig. 4a) on one end crossing the incident beam near the paraboloid (call this segment $\Sigma_{\text {in }}$ ) and on the other end crossing the focal plane $\left(\Sigma_{\text {out }}\right)$, one can establish the following relation:

$$
\iint_{\Sigma_{\text {in }}}\left(\mathbf{E}_{\text {in }} \times \mathbf{H}_{\text {in }}^{*}\right)_{\mathrm{z}} \mathrm{d} x \mathrm{~d} y=\iint_{\Sigma_{\text {out }}}\left(\mathbf{E}_{\text {out }}^{\prime} \times \mathbf{H}_{\text {out }}^{\prime_{z^{\prime}}} \mathrm{d} x^{\prime} \mathrm{d} y^{\prime},\right.
$$

where $\mathbf{E}$ and $\mathbf{H}$ inside the integral are harmonic components of the field and the primed quantities belong to the focal field in the $K^{\prime}$ reference frame.

Equation (30) can be rearranged using plane-wave decomposition, giving

$$
\begin{aligned}
& \iint_{\Sigma_{\text {in }}}\left(\left|E_{\mathrm{x}, \text { in }}\right|^{2}+\left|E_{\mathrm{y}, \text { in }}\right|^{2}\right) \mathrm{d} x \mathrm{~d} y=\gamma \\
& \quad \times \iint_{\Sigma_{\text {out }}}\left(\left|E_{\mathrm{x}^{\prime}, \text { out }}\right|^{2}+\left|E_{\mathrm{y}^{\prime}, \text { out }}\right|^{2}+\left|E_{\mathrm{z}^{\prime}, \text { out }}\right|^{2}\right) \mathrm{d} x^{\prime} \mathrm{d} y^{\prime} .
\end{aligned}
$$

Here, $\gamma$ is the expectation value of the direction cosine $m$ $\left(=\sqrt{1-p^{2}-q^{2}}\right)$ in the focusing beam. It is calculated by

$$
\gamma=\iint\left|\mathbf{E}_{\mathrm{in}, T}\right|^{2} m \frac{\partial(x, y)}{\partial(p, q)} \mathrm{d} p \mathrm{~d} q / \iint\left|\mathbf{E}_{\mathrm{in}, T}\right|^{2} \mathrm{~d} x \mathrm{~d} y .
$$

The value of $\gamma$ can be used to estimate the power ratio between transverse and longitudinal fields:

$$
\begin{aligned}
& \iint\left|E_{\mathrm{z}^{\prime}, \text { out }}\right|^{2} \mathrm{~d} x^{\prime} \mathrm{d} y^{\prime} / \\
& \iint\left(\left|E_{\mathrm{x}^{\prime}, \text { out }}\right|^{2}+\left|E_{\mathrm{y}^{\prime}, \text { out }}\right|^{2}\right) \mathrm{d} x^{\prime} \mathrm{d} y^{\prime} \approx 1-\gamma .
\end{aligned}
$$

Now let us consider momentum conservation, which can be deduced from combining the Lorentz force and the Maxwell equations [7]. This shows that the mechanical force is balanced by an electromagnetic force or a stress tensor. Using only time-averaged quantities leaves out the time derivative of the time-averaged field momentum and it becomes

$\left\langle F_{\text {mech }, \alpha}\right\rangle=\operatorname{Re} \oint \sum_{\beta} T_{\alpha \beta} n_{\beta} \mathrm{d} a$,

where $n_{\alpha}$ is a unit normal and $\mathrm{d} a$ is an infinitesimal area. The time-averaged Maxwell tensor, $T_{\alpha \beta}$, is

$$
\begin{aligned}
T_{\alpha \beta}= & \frac{1}{2} \epsilon_{0}\left(E_{\alpha} E_{\beta}^{*}-\frac{1}{2}|\mathbf{E}|^{2} \delta_{\alpha \beta}\right) \\
& +\frac{1}{2} \mu_{0}\left(H_{\alpha} H_{\beta}^{*}-\frac{1}{2}|\mathbf{H}|^{2} \delta_{\alpha \beta}\right) .
\end{aligned}
$$

Unfolding Eq. (34) under the condition that there is no longitudinal field in the incident beam in the geometry given, each force term in the $K^{\prime}$ frame is found as

$$
\begin{aligned}
\left\langle F_{\mathrm{x}^{\prime}}\right\rangle= & \operatorname{Re}\left[-\frac{1}{4} \epsilon_{0} \iint_{\Sigma_{\text {in }}} \sin \varphi\left(\left|E_{\mathrm{x}, \text { in }}\right|^{2}+\left|E_{\mathrm{y}, \text { in }}\right|^{2}\right) \mathrm{d} x \mathrm{~d} y\right] \\
& +\operatorname{Re}\left[\frac{1}{2} \epsilon_{0} \iint_{\Sigma_{\text {out }}}\left(E_{\mathrm{x}^{\prime}, \text { out }} E_{\mathrm{z}^{\prime}, \text { out }}^{*}\right) \mathrm{d} x^{\prime} \mathrm{d} y^{\prime}\right]+H \text { terms, } \\
\left\langle F_{\mathrm{y}^{\prime}}\right\rangle= & \operatorname{Re}\left[\frac{1}{2} \epsilon_{0} \iint_{\Sigma_{\text {out }}}\left(E_{\mathrm{y}^{\prime}, \text { out }} E_{\mathrm{z}^{\prime}, \text { out }}^{*} \mathrm{~d} x^{\prime} \mathrm{d} y^{\prime}\right)\right]+H \text { term },
\end{aligned}
$$




$$
\begin{aligned}
\left\langle F_{\mathrm{z}^{\prime}}\right\rangle= & \operatorname{Re}\left[-\frac{1}{4} \epsilon_{0} \iint_{\Sigma_{\text {in }}} \cos \varphi\left(\left|E_{\mathrm{x}, \text { in }}\right|^{2}+\left|E_{\mathrm{y}, \text { in }}\right|^{2}\right) \mathrm{d} x \mathrm{~d} y\right] \\
& +\operatorname{Re}\left[-\frac{1}{4} \epsilon_{0} \iint_{\Sigma_{\text {out }}}\left(\left|E_{\mathrm{x}^{\prime}, \text { out }}\right|^{2}+\left|E_{\mathrm{y}^{\prime}, \text { out }}\right|^{2}\right) \mathrm{d} x^{\prime} \mathrm{d} y^{\prime}\right] \\
& +\operatorname{Re}\left[\frac{1}{4} \epsilon_{0} \iint_{\Sigma_{\text {out }}}\left|E_{\mathrm{z}^{\prime}, \text { out }}\right|^{2} \mathrm{~d} x^{\prime} \mathrm{d} y^{\prime}\right]+H \text { terms. }
\end{aligned}
$$

Magnetic terms are obtained by simply replacing $\epsilon_{0}$ with $\mu_{0}$ and the $E$-vector with the $H$-vector, or one can simply multiply electric field terms by two and drop magnetic terms.

The force exerted on the mirror can be separately calculated using radiation pressure on the mirror. The pressure perpendicular to the mirror surface, if it is non-absorbing and the incident plane wave is at an angle $\theta$, is $\epsilon_{0}|\mathbf{E}|^{2} \cos ^{2} \theta$. Thus, in the $K$ frame the force is

$$
\langle\mathbf{F}\rangle=\iint_{\Sigma} \epsilon_{0}\left|\mathbf{E}_{\text {in }}\right|^{2} \cos ^{2} \theta \hat{\mathbf{n}} \mathrm{d} A,
$$

where $\cos \theta=\hat{\mathbf{n}} \cdot \hat{\mathbf{z}}=1 / \sqrt{1+s}$ and we use $\hat{\mathbf{n}}$ and $\mathrm{d} A$ from Eqs. (7) and (8). After rotating each $\langle\mathbf{F}\rangle$ component into the $K^{\prime}$ frame according to the rule in Eqs. (27)-(29), we can compare these values with the force components in the $K^{\prime}$ frame derived from the Maxwell stress tensors.

Applying these two numerical check procedures, we obtain a grid resolution dependent error which goes to zero at a higher resolution. Whether it is off-axis or on-axis or small numerical aperture or high numerical aperture, the numerical error in our diffraction formulae only depends on the size of the grid resolution. For example, the power error and the force error are about $1.5 \%$ at a $128 \times 128$ grid and $3 \%$ at a $64 \times$ 64 grid. We also note that with a smaller grid size one always underestimates the power density.

It is interesting to note that phase quadrature between the longitudinal field and the transverse field at the focus is clearly seen in Eqs. (36) and (37). Such phase-quadrature behavior has been known since Lax et al.'s higher-order differential solutions [8]. Suppose an on-axis case $(\varphi=0)$. As long as the illuminated mirror surface is symmetric, $\left\langle F_{\mathrm{x}^{\prime}}\right\rangle$ and $\left\langle F_{\mathrm{y}^{\prime}}\right\rangle$ are zero and consequently the phase of the longitudinal field has to be in quadrature with that of the transverse field. It may not be obvious, however, for off-axis focusing; for it is not clear how the force in the $x^{\prime}$ direction, now in action because of asymmetry, distributes itself among the energy term and the correlation term in Eq. (36). It can be shown by calculation that the phase quadrature still preserves its quality even at high NA with severe off-axis focusing (Fig. 4b): most of the asymmetric force arises from the energy term. From the experimental point of view, when on-axis focusing is hard to implement, this fact may be important especially for those who are concerned with a phase-sensitive experiment. On the other hand, one can always have a clear phase quadrature if one uses polarization along the $y$ axis. In addition to the experimental considerations, we mention that the longitudinal field does not enter in the calculation of peak power or peak intensity because at the peak of the transverse field oscillation the longitudinal field is always zero, which can be seen, as a result of phase quadrature, either from its spatial focal distribution [9] or time-domain picture [5]. Thus, in this article we define the peak value of the Poynting vector in the propagation direction as the peak intensity. If one uses plane-wave decomposition (Eq. (31)), it seems confusing that the longitudinal field is included in the calculation. The effect of it is almost nullified due to the $\gamma$ factor, which is smaller than 1 . In fact, the right-hand side of Eq. (31) can be approximated with summation of only transverse fields dropping $\gamma$ and the longitudinal term.

These criteria are not only useful in comparing with other vector diffraction theories [10-12] but also necessary to ensure the absolute exactness of numerical values. The latter point is especially important if one tries to calculate focused intensity directly from the diffraction integral. Compare this approach with a typical intensity estimation using encircled energy. If there is a halo in the focal spot distribution such as from amplified spontaneous emission, encircled energy in that case cannot well distinguish coherent contents from incoherent background while we are dealing with only coherent parts of the beam through a Shack-Hartmann sensor. Applying the criteria to the diffraction integrals in this article and to the theory of Richards and Wolf [10], one finds that both theories satisfy the first criterion whereas the second criterion is satisfied only by the present theory, which can be shown through numerical calculation.

Another miscellaneous point in the diffraction calculation has to be taken into account when the incident laser beam has a broad bandwidth. According to the analytic field expressions at the focus due to Rau et al., the effect of a short pulse on the calculation of intensity is only significant at the subcycle pulse-width regime [13]. In most cases the monochromatic diffraction formula is sufficient.

\section{3}

\section{Measurement of aberrations through imaging}

The field can be measured with a wavefront sensor of various kinds (e.g. Shack-Hartmann sensor, shearing interferometry) or with a phase-retrieval algorithm using only intensity profiles. Whichever measurement method is used, it is often necessary to relay the object field to the sensor area. The imaging of the field, however, inevitably introduces phase and intensity error at the sensor area. It is thus necessary to find conditions of imaging for which the field is truthfully copied from its original shape. Such a criterion was first proposed by Tichenor and Goodman [14]. This criterion says that the aperture size of the imaging optics has to be four times larger than the size of the object to prevent distortion of the field. This condition, however, seems too strong and in fact it immediately invalidates our wavefront-imaging setup, for our beam is slightly smaller than the paraboloid aperture, not four times. Nevertheless, the physical origin of the imaging error can be stated in a more clarified way and indeed keeping in mind such a physical picture helps us apply a correct imaging condition in a much more flexible way depending on the situations. Furthermore, it will be shown that our imaging system does not suffer from imaging degradation as was thought initially. In Tichenor and Goodman's experiment they performed a proofof-principle experiment that showed a severe image distortion when the imaging setup was not in accordance with their criterion. On careful examination of their experiment, 

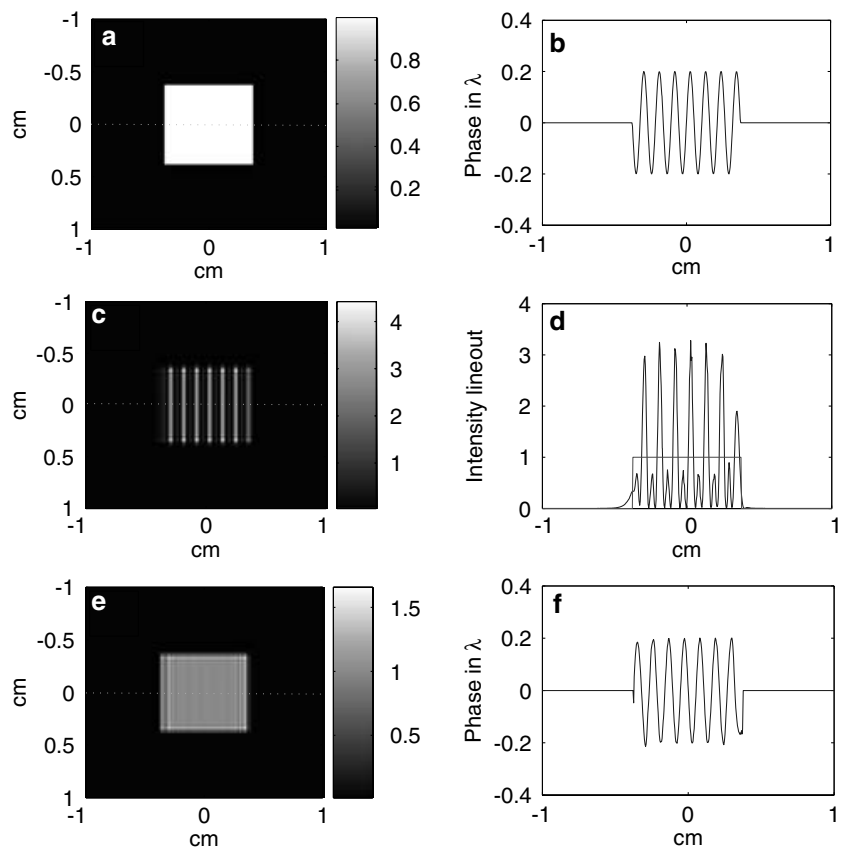

FIGURE 5 Imaging of wavefront. a Intensity profile at the object plane and b phase line-out of (a). c Intensity profile at the lens aperture and $\mathbf{d}$ line-out of the intensity profile with the lens aperture (rectangular line). e Intensity profile at the image plane and $\mathbf{f}$ phase line-out of the image

however, one realizes that the severely distorted imaged intensity profile is not so much from violating the condition as from the truncation of the incoming beam in the lens aperture. The lens aperture need not be so large, inasmuch as the incident beam does not exist near the edge of the lens. One can always find a situation where object size and lens aperture size are not much different while producing almost the same copy of the object field. This can be achieved if the object field does not contain rapid or deep modulations either in the phase or the amplitude, so that most of the propagated field at the lens aperture remains inside the aperture. One calculated example is shown in Fig. 5, where we use the same object size and focal length as used in [14]. In this example a 7.5-mm object with a sinusoidal phase was imaged through a $25-\mathrm{cm}$ focal length lens with a 1:1 imaging scheme using a He-Ne beam $(632.8 \mathrm{~nm})$. The lens aperture size is the same as the object size. The Fresnel number for the object field is 178 and the energy eclipsed by the lens aperture is $2 \%$. The field-propagation calculation was performed using the angular spectrum decomposition method [15] with Fresnel approximation: the input field is Fourier transformed into spatial frequency space and multiplied by a frequency-domain Fresnel kernel and then it is inverse Fourier transformed back to the spatial domain. The two-FFT approach is commonly used in diffraction calculation. Figure 5a and5e show object and image intensity distribution, respectively. The intermediate intensity distribution at the lens and its line-our along the dotted line are shown in Fig5c and5d. Figure 5b shows the sinusoidal phase profile of the object field and Fig. $5 \mathrm{f}$ shows the calculated phase profile at the image plane, which is inverted. The minor truncation on the left-hand side of the intensity at the lens aperture affects the imaged phase on the right, although the distortion is not much. Thus, a more relaxed criterion can be established by just imposing that the input intensity should not fill over the lens aperture. Even in the case of overfilling the lens aperture, the effect of truncation is only significant when the Fresnel number is small. For truncated beams of a large Fresnel number $\left(\sim 10^{4}\right)$, the imaged field is still a reliable duplication of the object field as long as we consider only the non-truncated portion of the beam.

4

\section{Experimental setup for measuring $f / 0.6$ paraboloidal aberrations}

\section{Experimental setup}

Figure 6 shows the experimental setup for the measurement of $f / 0.6$ paraboloidal aberrations (3-in diameter, 2 -in effective focal length). A 27-fs regeneratively amplified beam was used for the incoming beam. The laser system producing the regeneratively amplified beam will be described in Sect. 5. In this setup, the deformable mirror (DM) plane is imaged onto the wavefront sensor. As is depicted in Fig. 6, the beam edge is close to the boundary of the paraboloid; it, however, does not affect the quality of the imaged field as was explained in Sect. 3 because the Fresnel number is very large in this case. Since the diffraction effect in the beam is negligible for the short-distance propagation, the image of the deformable mirror through the paraboloid contains both beam aberrations and the paraboloidal aberrations linearly added together. The image formed by the paraboloid near the focus is relayed onto the Shack-Hartmann wavefront sensor (Imagine Optic) through the infinity-corrected, flat-field-corrected apochromatic objective $(\mathrm{NA}=0.75, \times 40)$ and an achromatic lens. Note that the objective forma a virtual image of DM, which means that the lateral position of the objective is slightly off from the position optimized for an infinity image. The object of this image is actually a tilted plane near the deformable mirror; however, the wavefront error from imaging the tilted plane must be negligible. The sensor has a $5 \mathrm{~mm} \times 5 \mathrm{~mm}$ capture area of 8-bit dynamic range with a $32 \times 32$ micro-lenslet array. The focal length and diameter of each microlens are $5.6 \mathrm{~mm}$ and $153 \mu \mathrm{m}$, respectively. The depth of focus is thus about $400 \lambda$, so the sensor is insensitive to the $45-\mathrm{nm}$ bandwidth of the 30 -fs pulse. The objective and the achromatic lens are aligned along the middle ray. The measured wavefront can be used to provide feedback to the deformable mirror or to predict the focal field distribution.

There might be a concern that the wavefront-imaging system cannot measure the longitudinal field. It can, however, be argued that, due to the time symmetry of the Maxwell equations in vacuum, the longitudinal field is absorbed back into transverse oscillations by the time the field reaches the wavefront sensor because the beam divergence is now significantly slower than the initial focusing.

\subsection{Correction algorithm}

First we explain the algorithm of finding optimum voltage solutions. Suppose the wavefront of the $k$ th actuator with unit voltage is expressed as $G_{i k}$, where index $k$ runs from actuator number 1 to 96 and index $i$ runs through each 


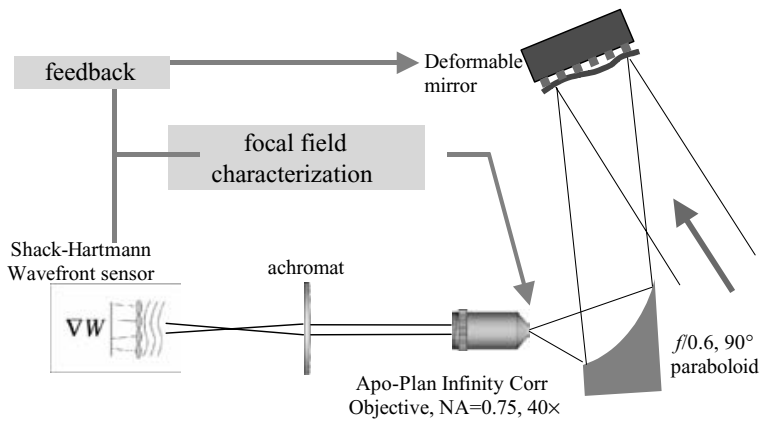

FIGURE 6 Experimental setup for measuring $f / 0.6$ paraboloidal aberrations

wavefront cell (e.g. a $32 \times 32$ microlens array makes $i=1$ 1024). Then, the wavefront $\left(\Phi_{i}\right)$ due to an arbitrary voltage distribution $\left(V_{k}\right)$ is

$\Phi_{i}=G_{i k} V_{k}$.

Let $W_{i}$ be the wavefront to be corrected and $\Phi_{0, i}$ be the objective wavefront, which is usually all zero. The optimum voltage distribution is calculated by

$\mathbf{V}=\mathbf{G}^{-1}\left(\Phi_{0}-\mathbf{W}\right)$.

The inverse of the non-square matrix $\mathbf{G}$ can be calculated with the singular value decomposition method. The performance of this setup depends on the aberrations of the receiving optics (the objective and the achromatic lens) and the statistical stability of the wavefront.

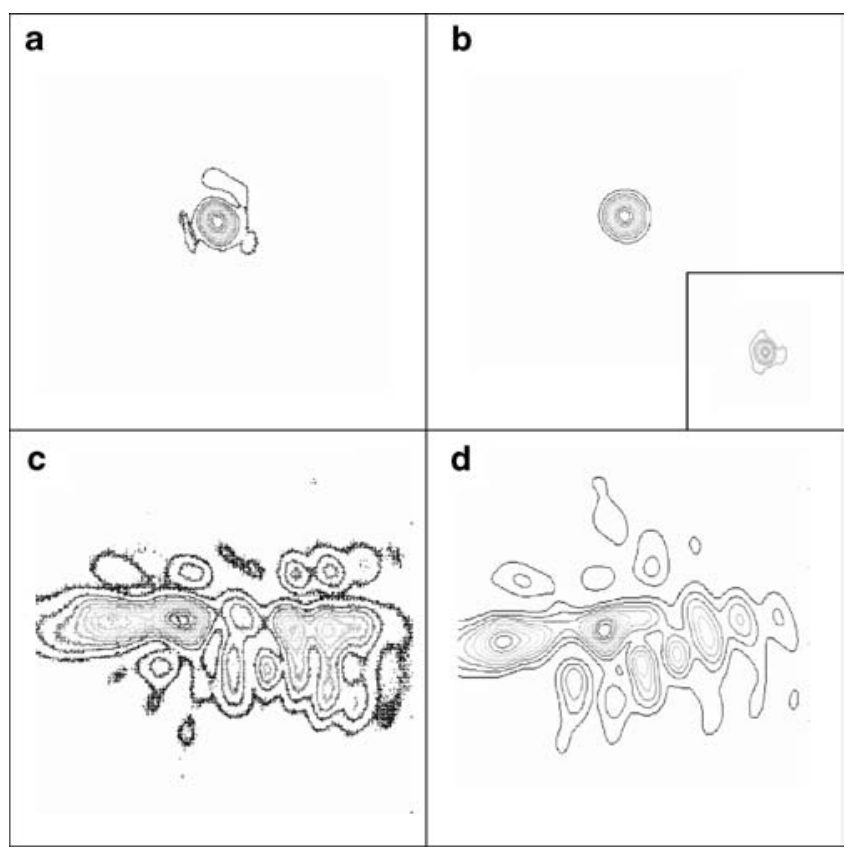

FIGURE 7 Comparison of focal spot calculation $(\mathbf{b}, \mathbf{d})$ and measurement with a 12-bit CCD camera (a, c) using the regeneratively amplified beam. Before correction $(\mathbf{c}, \mathbf{d})$ and after correction $(\mathbf{a}, \mathbf{b})$. b and $\mathbf{d}$ were calculated by using measured phase and amplitude profiles after focusing. The inset in (b) is a calculated focal spot at $1 \mu \mathrm{m}$ away from the focus, which more likely matches with (a)
The effect of receiving optic aberrations can be considered negligible if the calculated focal field distribution matches well with the measured focus picture. We calculated the field distribution at the focus using the formulae (20)-(26) and compared the calculated images with the experimental focal pictures measured with a 12-bit CCD camera (Fig. 7). Figure $7 \mathrm{a}$ shows the measured corrected focal spot with $0.8 \mu \mathrm{m}$ full width at half maximum. The resolution of the image is 40 pixels per micrometer. The region of the focal spot (Fig. 7a) where the local intensity is larger than half the peak intensity contains $30 \%$ of the total energy, whereas its calculated counterpart (Fig. 7b) contains $44 \%$. Besides the errors due to background correction, the discrepancy may be attributed to the sensitivity of the encircled energy of the experimental picture to defocusing error. In this tight-focusing configuration, for example, $1-\mu \mathrm{m}$ defocusing results in $27 \%$ reduction in the encircled energy (Fig. 7b, inset). Note also that there can be about $10 \%$ error in the encircled energy due to the receiving optic aberrations (please refer to Sect. 5). Assuming we could make $10 \%$ error each in background correction, focusing image, and imaging aberrations, it seems that $30 \%$ discrepancy is within a reasonable range. There will, however, always be a fundamental discrepancy between calculated spot and measured spot because the wave-front measurement excludes the incoherent focal spot halo as was mentioned earlier. The calculation and measurement comparison for the uncorrected focal spot is presented in Fig. 7c and 7d. The application of this setup in a 45-TW laser system to achieve an ultra-high-power-density focused beam is presented in Sect. 5.

\section{5}

\section{Generation of $10^{22}-\mathrm{W} / \mathrm{cm}^{2}$ power density}

The aberration measurement and correction system presented above cannot be directly used at high power because the objective will be damaged. One can however obtain a nearoptimum result if a low-energy reference beam is available, which is close to the main beam in its wavefront shape.

The all-Ti:sapphire laser system used in this experiment consists of a Kerr-lens mode-locked oscillator, a regenerative amplifier $(40 \mathrm{~mJ})$, and a four-pass amplifier $(600 \mathrm{~mJ})$ followed by a two-pass amplifier $(6.7 \mathrm{~cm} \times 5.4 \mathrm{~cm}$ elliptical beam, $2.5 \mathrm{~J})$ [16]. Pulses are stretched to 350 ps before amplification [17] and compressed to $30 \mathrm{fs}$ at 10-TW level, which was measured by a tilted pulse-front single-shot autocorrelator [18]. The throughput of the compressor is 55\% and the chamber is pumped down to $5 \times 10^{-5}$ Torr. The bandwidth of the regenerative amplifier is $45 \mathrm{~nm}$ and it is reduced to $43 \mathrm{~nm}$ at $30 \mathrm{TW}$. For this laser system the regenerative-amplifier beam can be used as a reference beam since the wavefront of the regenerative-amplifier beam relative to the 45 -TW beam is only $0.123 \mu \mathrm{m}$ in the root-mean-square (r.m.s.) sense. The relative wavefront was measured with a 10:1 telescope through a high-reflectivity mirror placed just before the deformable mirror. The reflectivity of the mirror is high enough (transmission is about $10^{-3}$ ) so that the nonlinear effect through the mirror substrate at $45 \mathrm{TW}$ is negligible. The aberrations of the telescope do not enter in the 45-TW relative wavefront 

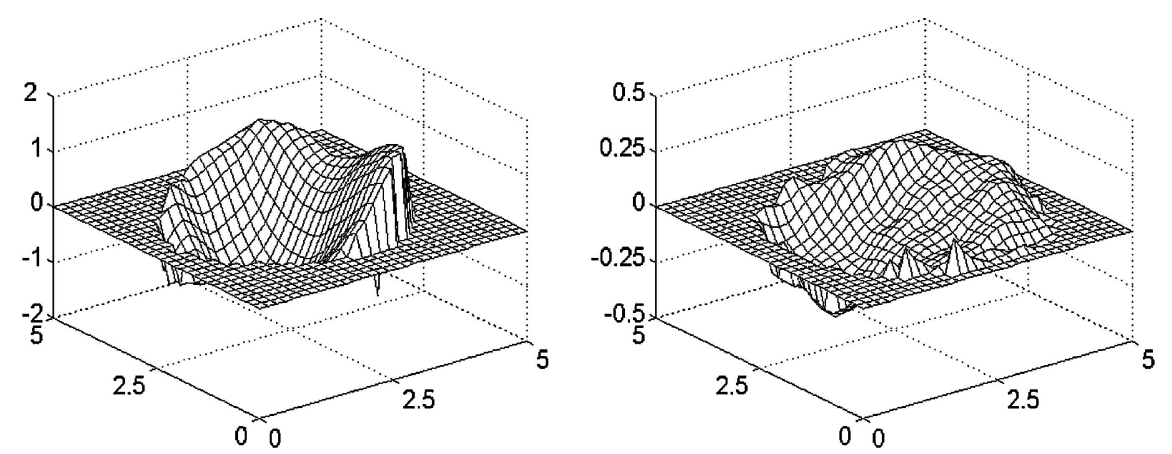

(a) r.m.s. $=0.936 \lambda$, Peak-to-Valley (P.V.)

(b) r.m.s. $=0.154 \lambda$, P.V. $=0.657 \lambda .=4.247 \lambda$.

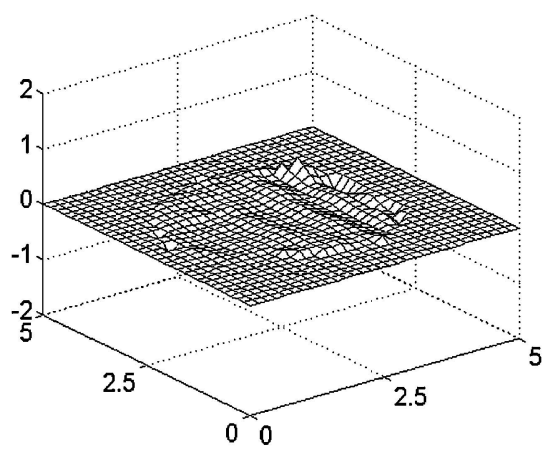

(c) r.m.s. $=0.154 \lambda$, P.V. $=0.657 \lambda$.

FIGURE 8 Wavefronts of a regenerative-amplifier beam after paraboloid, b 45-TW beam against regenerative-amplifier beam, $\mathbf{c}$ corrected regenerativeamplifier beam after paraboloid

measurement, for they are subtracted out in relative measurement mode. The direct use of a regenerative-amplifier beam as a reference beam is still not possible until the energy of the beam is attenuated through neutral-density filters. These filters introduce non-negligible tilt error and negligible higher-order aberrations (r.m.s. $\sim 0.015 \lambda$ ). The tilt error can be easily corrected by using an external pointing system.

The wavefront of the regenerative-amplifier beam after the paraboloid and the relative wavefront of the regenerativeamplifier beam against the 45-TW beam are shown in Fig. 8a and b. The wavefront of the regenerative-amplifier beam after the adaptive optic correction loop is shown in Fig. 8c. The 3-in deformable mirror (Xinetics, Inc.) has 97 lead-magnesium-niobate actuators $( \pm 2-\mu \mathrm{m}$ interactuator stroke). The actuators exhibit a linear response to the applied voltage due to the electrostriction effect. In this experiment the voltage was operated at around $50 \mathrm{~V}$. The mirror surface is coated with protective silver with a damage threshold of $300 \mathrm{~mJ}$ measured with 30 -fs pulses.

Using the measured wavefront and also the amplitude profile, which was measured separately, we calculated the peak intensity of a 1.4-J, p-polarized (along $x$ axis), 30fs pulse focused by the $f / 0.6$ paraboloid using Eqs. (20)(22) in three cases, assuming no interaction with material in $5 \times 10^{-5}$ Torr. Figure 9a shows the focused intensity distribution without any corrections $\left(I_{\text {peak }}\right.$ (peak intensity) $=1 \times 10^{21} \mathrm{~W} / \mathrm{cm}^{2}$ ) and Fig. 9b corresponds to the case when only reference beam and paraboloidal aberrations were corrected $\left(I_{\text {peak }}=(6.9 \pm 0.7) \times 10^{21} \mathrm{~W} / \mathrm{cm}^{2}\right)$. In principle, it is possible to obtain an even higher peak intensity of $(1 \pm 0.1) \times 10^{22} \mathrm{~W} / \mathrm{cm}^{2}$ (Fig. 9c) as the following. If $\Phi_{0}$ in Eq. (41) was the negative value of the relative wavefront in Fig. 8b, we could have obtained the optimum voltage distribution for a $45-\mathrm{TW}$ beam rather than for the regenerative beam. In such a differential correction mode, $\Phi_{0}$ has to be converted according to the transformations of Eqs. (12), (16), and (17) before it is inserted into the correction algorithm (Eq. (41)). We demonstrated such differential correction with a He-Ne beam using the same geometry as used in this experiment. The differential wavefront error of a thin glass plate was separately measured and inserted into the correction algorithm. The aberration of the thin plate can be thought of as simulating a high-energy differential phase. The wavefront after the paraboloid was improved from $0.23 \lambda$ r.m.s. without the plate to $0.12 \lambda$ r.m.s. with the plate inserted. The focal spot size was reduced by $15 \%$. The performance could not be improved further due to a secondary-reflection from the plate.

The error range of the calculated peak intensity was estimated from an approximate Strehl ratio formula, $S=$ $\exp \left(-2 \pi \sigma^{2}\right)$, which works well with small aberration. The error of the wavefront-imaging optics is estimated to be $0.02 \lambda$ r.m.s. This estimation is based on the measurement of a wavefront emerging from a single-mode fiber tip through similar imaging optics (a simple infinity-corrected objective of $\times 40$ magnification with NA $=0.66$ and a plano-convex lens). In 

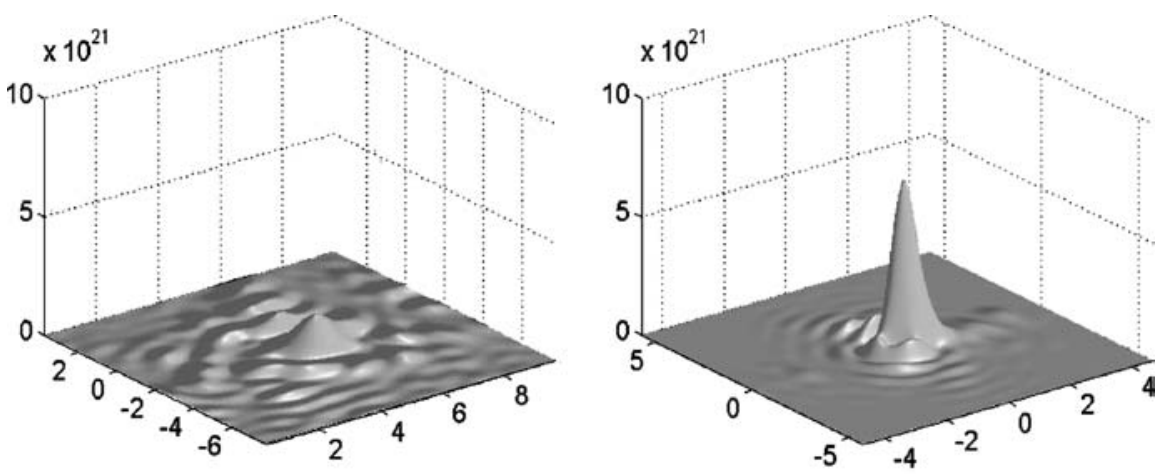

(a) $\mathrm{I}_{\text {peak }}=1 \times 10^{21} \mathrm{~W} / \mathrm{cm}^{2}$

(b) $\mathrm{I}_{\text {peak }}=(6.9 \pm 0.7) \times 10^{21} \mathrm{~W} / \mathrm{cm}^{2}$

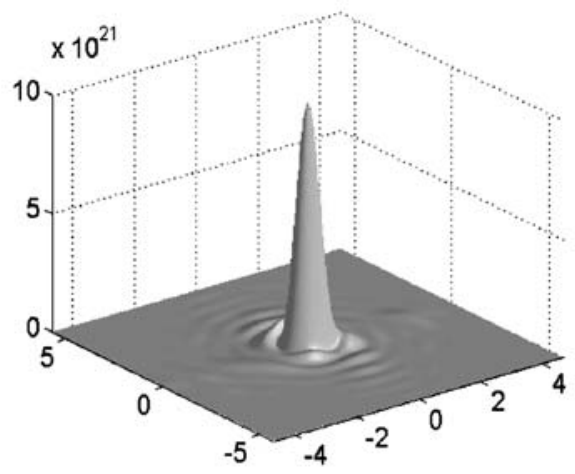

(c) $\mathrm{I}_{\text {peak }}=(1 \pm 0.1) \times 10^{22} \mathrm{~W} / \mathrm{cm}^{2}$

FIGURE 9 Calculated power-density profile of 45-TW focused beam a without correction, $\mathbf{b}$ with correction of the reference-beam aberrations and the paraboloidal aberrations only, $\mathbf{c}$ with correction of all aberrations including 45-TW relative aberration

addition to a measurement error bar, one can include the effect of statistical fluctuations (r.m.s.) in the wavefront. The statistical r.m.s. values are calculated by averaging the r.m.s. value of each wavefront relative to the mean wavefront shape using 10-20 shots. The statistical r.m.s. values were measured to be $0.04 \lambda$ at the regenerative and four-pass amplifiers and $0.06 \lambda$ at the two-pass amplifier level ( $>30 \mathrm{TW})$. The range of errors will generally increase if the statistical effect is included although the measurement error and the statistical error are of different natures. In our error estimation we included only measurement error because the field calculation and the intensity profile are based on one-shot measurement, although it is not entirely true single shot since weak-energy measurement averages over many shots.

\section{6}

\section{Conclusion}

We obtained far-field vector diffraction formulae in Fourier-transform format for an off-axis paraboloid using the Stratton-Chu theory and developed an experimental method for measuring the aberrations of a high-NA optic. It was shown that the measured wavefront can first be utilized for aberration correction using a deformable mirror and then inserted into the diffraction calculation to obtain the phase and amplitude at the focus. This method was proven to be effective in generating and characterizing ultra-high electromagnetic field intensity. Such a high intensity can be used to study high-field laser-matter interactions [19]. It can also be applied to generate a special form of focal field by defining a corresponding objective field in Eq. (41).

ACKNOWLEDGEMENTS This work was supported by the National Science Foundation through the Physics Frontier Centers Program at the University of Michigan with Grant No. PHY-0114336.

\section{REFERENCES}

1 O. Albert, H. Wang, D. Liu, Z. Chang, G. Mourou, Opt. Lett. 25, 1125 (2000)

2 S.-W. Bahk, P. Rousseau, T.A. Planchon, V. Chvykov, G. Kalintchenko, A. Maksimchuk, G.A. Mourou, V. Yanovsky, Opt. Lett. 29, 2837 (2004)

3 P. Varga, P. Török, J. Opt. Soc. Am. A 17, 2081 (2000)

4 L. Cicchitelli, H. Hora, R. Postle, Phys. Rev. A 41, 3727 (1990)

5 B. Quesnel, P. Mora, Phys. Rev. E 58, 3719 (1998)

6 S.-W. Bahk, V. Yanovsky, G. Mourou, in Conference on Lasers and Electro-Optics (CLEO), 2005, paper JThE84

7 J.D. Jackson, Classical Electrodynamics, 2nd edn. (Wiley, New York, 1975), p. 242

8 M. Lax, W.H. Louisell, W.B. McKnight, Phys. Rev. A 11, 1365 (1975)

9 R. Dorn, S. Quabis, G. Leuchs, J. Mod. Opt. 50, 1917 (2003)

10 B. Richards, E. Wolf, Proc. R. Soc. (Lond.) A 253, 358 (1959)

11 W.H. Carter, J. Opt. Soc. Am. 62, 1195 (1972)

12 R.K. Luneberg, Mathematical Theory of Optics (Brown University, RI), mimeographed lecture notes (1944) 
13 B. Rau, T. Tajima, H. Hojo, Phys. Rev. Lett. 78, 3310 (1997)

14 D.A. Tichenor, J.W. Goodman, J. Opt. Soc. Am. 62, 293 (1972)

15 J.W. Goodman, Introduction to Fourier Optics, 2nd edn. (McGraw-Hill, New York, 1996), Sect. 3.10

16 V. Yanovsky, S.-W. Bahk, C. Felix, N. Saleh, P. Rousseau, V. Chvykov, G. Mourou, in Conference on Lasers and Electro-Optics (CLEO). OSA Trends in Optics and Photonics Series, vol. 73 (Optical Society of America, Washington, DC, 2002), paper CMK4
17 P.S. Banks, M.D. Perry, V. Yanovsky, S.N. Fochs, B.C. Stuart, J. Zweiback, IEEE J. Quantum Electron. 36, 268 (2000)

18 Z. Sacks, G. Mourou, R. Danielius, Opt. Lett. 26, 462 (2001)

19 S.V. Bulanov, T.Zh. Esirkepov, J. Koga, T. Tajima, Plasma Phys. Rep. 30, 196 (2004) 